\title{
Editorial
}

\section{Procesos de fronterización y desfronterización en territorios latinoamericanos}

\author{
NanetteLiberona Concha \\ Universidad Arturo Prat, Iquique, Chile \\ Email: nliberonac@gmail.com \\ Camila Álvarez Torres \\ Universidad Arturo Prat, Iquique, Chile \\ Email: camalvarez@unap.cl \\ Gabriela Córdova - Rivera \\ Universidad de Los Lagos, Santiago, Chile. \\ Email: gabriela.cordova@ulagos.cl
}

El auge de la globalización en los años 1990 hizo plantear a muchos intelectuales la idea del fin de las fronteras, algunos más aventureros expresaron que entrábamos en una "era postcolonial”, en la cual el Estado-nación era fuertemente cuestionado (Appadurai, 1996). Sin embargo, desde entonces hemos sido testigos del "retorno de las fronteras" (Amilhat-Szary, 2006). Con dolor e impotencia hemos visto la multiplicación de muros fronterizos, más de treinta muros desde el 11-S, quince solamente en el 2016 (Moras, 2016). Este fenómeno denominado “fronterización” (Grimson, 2003) ha significado una transformación de las fronteras, cumpliendo más bien la función de "filtro de flujos" para llevar a mejor término un tratamiento diferenciado de los mismos (Amilhat-Szary, 2015; Liberona, 2015). A pesar de que este fenómeno no sólo atañe a la migración, ésta ha sido la principal afectada, debido al aumento de la migración irregularizada y por tanto de los cruces clandestinos. Álvarez (2016) afirma que el ingreso clandestino a países como Estados Unidos y los europeos, ha sido extremadamente perseguido en los últimos años, sin lograr disminuir el flujo de migrantes indocumentados, sino más bien atrayendo al crimen organizado, que ha logrado controlarlo a través de las tarifas y de los peligros mortales (Álvarez, 2016; Kearney, 2006). En la actualidad, este tipo de migración se expande en Latinoamérica, pues las crisis económicas y políticas de países como Colombia, Cuba y Venezuela han transformado las rutas migratorias tradicionales, dirigiéndose a países vecinos o del sur del continente, demostrando a su vez que las estructuras rígidas alimentan fenómenos como el tráfico y la trata de personas (Rodríguez, 2007; Bello, 2013). 
En América latina, las fronteras tienen la característica en común de que fueron impuestas para "ordenar" el Nuevo Mundo (Popescu, 2011). Esto demuestra el carácter exógeno de la construcción de las fronteras latinoamericanas, así como un ordenamiento del mundo de tipo colonial y occidental que se aleja de la comprensión local del mismo, lo que incide en su construcción, deconstrucción y reconstrucción. Otra de las características relevantes es que en la mayoría de los casos las fronteras están situadas en lo que se denomina "zonas extremas", es decir, alejadas de los centros, con baja población y bajos niveles de desarrollo respecto a los mismos países (AmilhatSzary y Rouvière, 2009). Estas características las convierten en espacios que muchas veces han sido considerados "vacíos", concepción que ha sido propicia para la intervención estatal de las fronteras, sin considerar las realidades e interacciones locales. Sin embargo, los dinamismos propios de las fronteras nos invitan a plantear fenómenos que podríamos llamar de “desfronterización”, de apertura o de transgresión de los límites impuestos, a pesar de que a veces se enmarcan en contextos de consolidación de los Estados-nacionales. Esto ha podido observarse a partir del desarrollo fronterizo experimentado, es decir, de las condiciones socio-económicas que afectan de manera particular a la sociedad local (Dilla y Álvarez, 2018), por ejemplo a la "ciudad de frontera", sus dinámicas de intercambio y los diversos proyectos políticos de desarrollo fronterizo, tal como relatan los autores Camuss y Rossenblit (2011) sobre la ciudad fronteriza de Arica, en el norte de Chile, durante la primera mitad del siglo XX.

Es importante que, dentro de un contexto global de aumento de teorías en los estudios fronterizos, desde nuestro Sur, reunamos al pensamiento emergente sobre el tema. Y este es el objetivo que buscó el llamado de este número; recoger desde un lente de aproximación "multifocal” las interpretaciones que se están haciendo actualmente respecto del problema de la fronterización/desfronterización de acuerdo a las especificidades locales de las fronteras de la región. No obstante, en el presente número, los artículos recibidos, y los seleccionados, se posicionan desde prácticamente dos grandes paradigmas sobre la frontera; por un lado, el impacto de la fronterización en las migraciones, situando el debate de la frontera, no solamente como límite jurídico-administrativo de los países, sino también como fundamento simbólico de las políticas públicas que atañen a la inmigración en Chile y Sudamérica. Y, por otro lado, la dinámica transfronteriza que se da en el espacio fronterizo, definido como multidimensional y multiescalar, la que constituye diversas territorialidades. En el primer caso, en el discurso y las acciones políticas, la soberanía del Estado es argumento para vulnerar los derechos de los y las migrantes. En el segundo caso, la soberanía estatal ha sido fundamento para construir territorialidades que desconocen o invisibilizan la transfrontericidad, especialmente en su dimensión económica, en desmedro de las regiones fronterizas y los actores que ahí se relacionan.

El primer texto del dossier lo inaugura el artículo de Haroldo Dilla e Ingrid Breton con "Las regiones transfronterizas en América Latina". Los autores analizan desde una perspectiva teórica y latinoamericana, los debates actuales sobre las regiones transfronterizas. En primer lugar, se 
cuestiona la percepción binaria del ampliamente estudiado dilema de la contraposición (re)fronterización/desfronterización, en tanto se trataría más bien de un proceso sincrónico que contiene la relación contacto/separación. En segundo lugar, se plantea que se ha dejado de lado la esencia del problema, que es la manera en que las fronteras internacionales intermedian en los procesos de acumulación económica, y los articulan, reproduciendo el poder político y el funcionamiento del sistema. Luego, los autores centran su análisis en las regiones transfronterizas (RTF), las que definen--siguiendo a Jessop (2001)- como una articulación espacial multidimensional y multiescalar; sistemas espacio/temporales que abarcan territorios contiguos bajo jurisdicciones nacionales diferentes. Utilizadas como "laboratorios institucionales neoliberales" o como filtros para garantizar "los procesos de intercambio desigual”, las RTF, diferenciadas en cuatro tipologías por los autores, serían territorialidades que han constituido escenarios de valorización del capital a diferentes escalas.

El segundo artículo "Incivilidades". Notas sobre cómo la intervención estatal nocturna en el centro de Antofagasta endurece "las fronteras" recreando la fantasía de la inseguridad” es de autoría de Clive Echagüe, quien tuvo como propósito analizar los actos performáticos de securitización del Estado sobre los migrantes en el centro de la ciudad de Antofagasta, ubicada en la región de su mismo nombre al norte de Chile. Desde un enfoque que rescata el concepto de "incivilidades" como categoría de racialización, el autor analiza la reproducción de las percepciones sobre lo civil y lo incivil, entre lo humano y lo infrahumano y la generación de políticas públicas que profundizan la asociación entre migración, crimen y seguridad. A partir del uso de la técnica etnográfica y la revisión de fuentes secundarias, el autor señala que la intensa propaganda y relatos sostenidos por diversos actores estatales y sectores de la sociedad lograron instalar en la comunidad antofagastina un fuerte discurso de inseguridad, que evoca la idea de "incivilidades", frente a la llegada de migrantes y su uso del centro de la ciudad. Proyectos como el "Plan Centro Seguro" (PCS), en el cual el autor centra su análisis, se presentan como parte de los programas estatales que orientaron la percepción social de la inseguridad en un juego que tuvo como objetivo instalar "fronteras": la reproducción del miedo o, como señala el mismo texto, "pánico moral” en torno a ese espacio.

En el tercer artículo, “La Zona Franca de Iquique y su impacto en el trabajo informal de mujeres migrantes fronterizas” las autoras- Luz Icarte, Natalia Torres y Romina Ramos- analizan desde un enfoque que cruza las categorías de género, mercado laboral y migración fronteriza, el caso particular de las condiciones laborales de las mujeres migrantes en el espacio comercial-laboral de la Zona Franca situada en la ciudad de Iquique, región de Tarapacá, norte de Chile. Esta región tiene fuertes vínculos transnacionales, comerciales y migratorios con el centro oeste sudamericano, especialmente con países como Bolivia. A partir de un intenso trabajo de campo, las autoras señalan que la vinculación histórica, ininterrumpida en el tiempo, y fronteriza entre ambas regiones, ha permitido que la Zona Franca, dispositivo creado en la década de los setenta como motor de desarrollo 
regional, se convirtiera en un espacio de recepción de mano de obra feminizada, generando un mercado laboral circulatorio que ofrece tipos de trabajos altamente precarizados, irregulares e informales, que afectan principalmente a mujeres fronterizas. De manera particular, las autoras analizaron que las relaciones laborales que se desarrollan en ese espacio predispone a la mujer únicamente como sujeto productivo, en tanto, sus empleadores les niegan la posibilidad de realizar gestiones administrativas que les permitan ser sujetas de derecho, en el ámbito migratorio y laboral El texto de Luis Andrés Iturra, “La frontera tarapaqueña y sus territorialidades desde la crisis salitrera hasta la década dorada (1919-1960)”,se posiciona teóricamente en la interfaz entre la perspectiva reflectivista de los estudios en Relaciones Internacionales y la Geografía crítica, para analizar la frontera tarapaqueña y las distintas lógicas territoriales de sus actores subnacionales durante la crisis terminal del ciclo del salitre. De manera particular, el autor se centra en comprender la trayectoria que tuvieron los distintos imaginarios territoriales de diversos actores subnacionales y sus respectivas escalas de acción sobre el ámbito del poder y la configuración del espacio fronterizo, particularmente en relación al Estado chileno que actúo como dispositivo hegemónico de soberanía estatal. La recopilación bibliográfica y el uso de fuentes primarias permitieron reconstruir, según el autor, la existencia de tres territorialidades tarapaqueñas que, en un fuerte diálogo y contacto con sus vecinos de la región de Oruro en Bolivia, lograron desbordan la "trampa territorial”" del Estado, especialmente al generar una territorialidad que no dejó de lado la dinámica transfronteriza; enriqueciendo aún más el espacio fronterizo de Tarapacá.

El quinto artículo centrado también en la zona norte del país, está referido a la tensión entre el principio de soberanía de los estados y los derechos humanos de las personas migrantes, vulnerados en el control fronterizo. IskraPavez-Soto y Sofía Colomés, presentan “Derechos humanos y política migratoria. Discriminación arbitraria en el control de fronteras en Chile”. Mediante análisis documental y entrevistas, las autoras indagan en los vacíos legales y en las garantías jurídicas que existen en diversos instrumentos legislativos y proyectos de ley sobre migraciones. Identifican falencias vinculadas al respeto de los derechos humanos, debido al vacío legal y la descoordinación institucional en la legislación actual, en los tratados internacionales firmados y/o ratificados, y en los proyectos de ley. Las autoras concluyen que el hecho de impedir la entrada de ciertas personas migrantes se debe al carácter policial y de control que tiene la actual legislación y a la discrecionalidad en la aplicación de instrumentos jurídicos. Esta discrecionalidad en las funciones de control fronterizo (como la exigencia de solvencia económica y el no reconocimiento de las solicitudes de refugio, por mencionar algunas), se ampara en la ausencia de una política migratoria explícita que más allá de contemplar y ofrecer una solución efectiva al vacío legal que facilita la discriminación arbitraria, la perpetúa.

En el texto "La construcción política de la frontera. Entre los discursos nacionalistas y la 'producción' de trabajadores precarios”, Carolina Stefoni, Menara Guizardi y Herminia Gonzálvez analizan la contingencia 
de las políticas migratorias adoptadas por el gobierno de Chile hacia la población venezolana y haitiana durante el 2018, a partir del concepto de fronterización. Las políticas de control de las fronteras en gran parte del continente, tienden a favorecer el desarrollo de la economía global neoliberal y la reproducción de discursos nacionalistas que instalan la idea de que la migración amenaza las identidades. De esta forma, dichas políticas de control van produciendo, por un lado, "candidatos perfectos para los mercados laborales precarizados", sujetos migrantes en condiciones de vulnerabilidad extrema y por otro, una solución posible "frente a la idea construida de amenaza que sostienen los discursos nacionalistas". La selectividad y cierre nos dirán las autoras, operan como mecanismos de fronterización, mientras la selectividad caracteriza la gestión migratoria dirigida a la población venezolana, (visa de responsabilidad democrática), el cierre y restricción es propio de la visa consular dirigida al flujo de personas haitianas. Tras el lema de "migración segura, ordenada y regular" se incrementan los controles migratorios, el cierre selectivo y la implementación de discursos de criminalización y rechazo de los migrantes. De aquí la importancia de las fronteras como un "locus prioritario para estudiar los procesos de construcción de los macro modelos políticos y económicos, así como la identidad”.

Finalmente, Carina I. Trabalón, con su artículo "Política de visado y regulación de las fronteras. Un análisis desde la movilidad de haitianos en Sudamérica”, aborda la triada movilidad, fronteras y seguridad con relación al recorrido histórico de la reciente migración haitiana en América del Sur, específicamente en Brasil, Argentina y Chile. Mediante el análisis documental Trabalón profundiza en el estudio de las "condiciones de emergencia, efectos prácticos y fundamentos a través de los cuales se implementa la política de visado" orientada a este colectivo en dichos países. La autora identifica algunos elementos transversales en los tres países, que trascienden las particularidades de los marcos nacionales y sus normativas específicas. En torno a la migración haitiana, surgen una serie de categorías clasificatorias que activan mecanismos de inclusión/exclusión que, de acuerdo a los “diferentes contextos históricamente situados”, cuestionan el vínculo entre movilidad, fronteras y seguridad. Al igual que en la entrega anterior, entre los resultados de este artículo se identifican prácticas estatales restrictivas y selectivas que operan en los cruces de frontera de los tres casos estudiados, lo que -bajo argumentos securitarios y humanitarios de control- sitúa al "visado como mecanismo fundamental para la regulación de los movimientos migratorios a través de las fronteras".

Es satisfactorio ver cómo en estos artículos aparecen temas contingentes, tales como el cuestionamiento a las políticas de "seguridad" y de "control” vinculadas a la inmigración o el auge de la migración haitiana en Sudamérica; en los que se entrecruzan categorías de análisis, como género, racialización, trabajo, posicionando por ejemplo, la incidencia de la migración fronteriza femenina en la migración sur-sur y en el mercado laboral. Como podemos apreciar en este dossier, el norte de Chile (Antofagasta, 
Arica y, en especial Tarapacá), se presenta como un laboratorio de estudio y sobre fenómenos fronterizos y transfronterizos. Es indudable que la producción académica de estas latitudes ha impactado la producción nacional sobre migración y frontera.

En síntesis, este número recopila textos que evidencian las complejas y distintas realidades de las fronteras en Chile y Latinoamérica, a partir de los procesos de fronterización y de las dinámicas transfronterizas. Los impactos que éstos tienen en los derechos de las personas migrantes, en la migración sur-sur, en el desarrollo fronterizo, ponen a las fronteras en el centro de la discusión y en el relato de los Estados contemporáneos, como también en el ojo del análisis de las ciencias sociales en general, siendo este número una contribución al debate. 


\section{Bibliografía}

Álvarez, S. (2016). ¿Crisis migratoria contemporánea? Complejizando dos corredores migratorios globales. Ecuador Debate 97: 155-171.

Amilhat, A-L, y Fourny, M.C. (eds). (2006). Après les frontières, avec la frontière. Nouvellesdynamiquestransfrontalières en Europe. Paris, France :Editions de l'Aube.

(2009). Des dynamiquestransfrontalièresaubiland'aménagement du territoire: Innovations et blocagesdans les Andes centrales (Chili-PérouBolivie). ReveuMosella, $N^{\circ}$ Especial «Fronteras y desarrollo».

(2015). Qu'est-ce qu'unefrontièreaujourd'hui?, France, Paris :PressesUniversitaires de France.

Appadurai, A. (2005). Après le colonialisme. Paris, Francia: PetitebibliothèquePayot

Bello, D. (2012). Alianza Estratégica Aymaras sin Fronteras: Una respuesta territorial a los desafíos de la “glocalización. Tinkazos, 15(32), 147-164.

(2013). La Triple Frontera como polo de atracción de actividades ilícitas: Condiciones endógenas institucionales e ilegalidad. Atenea (Concepción), (508), 101-120.

Camus P., Rosenblit, J. (2011). Aislamiento de la frontera norte de Chile: ¿problema u oportunidad? En F. Arenas, A. Núñez y A. Salazar (eds.), El aislamiento geográfico: ¿problema u oportunidad?, (pp.59 -73). Santiago, Chile: PUCCH

Dilla, H. y Álvarez, C. (2018). Economía e intercambio desigual en una región transfronteriza: Arica, Chile-Tacna, Perú. Estudios fronterizos, 19, Recuperado de: http://ref.uabc.mx/ojs/index.php/ref/article/ view/670/1386

Dilla, H. (2015). Los complejos urbanos transfronterizos en América Latina.Revista de Estudios Fronterizos, Nueva Época, 16 (31) 15-38.

(2008). Las ciudades en las fronteras: introducción a un debate.en H. Dilla (ed.), Ciudades en las fronteras. Aproximaciones críticas a los complejos urbanos transfronterizos. (pp. 17-29) Santo Domingo, República Dominicana: Editora Manatí.

González, S., Rouviere, L. y Ovando, C. (2008). De “Aymaras en la frontera” a "Aymaras sin fronteras": los gobiernos locales de la triplefrontera andina (Perú, Bolivia y Chile) y la globalización. Diálogo andino / Universidad de Tarapacá, (31), p. 31-46. 
Grimson, A. (2003). Los procesos de fronterización: flujos, redes e historicidad. En García, C. I. (Comp.) Fronteras, territorios y metáforas,(pp 15-33), Medellín, Colombia: Hombre Nuevo Editores.

Hevilla C. y Zusman, P. (2007). Movilidades y construcción de nuevas territorialidades en la frontera chileno-argentina. Scripta Nova. Revista electrónica de Geografía y Ciencias Sociales. 11 245(22)

Kearney, M. (2006). El poder clasificador y filtrador de la frontera. En Besserer, F. y M. Kearney (editores) San Juan Mixtepec una comunidad transnacional ante el poder clasificador y filtrador de la frontera. Ciudad de México, México: Universidad Autónoma Metropolitana de México.

Liberona, N. (2015). La frontera cedazo y el desierto como aliado. Prácticas institucionales racistas en el ingreso a Chile. Polis Revista Latinoamericana, (42)143-165, Recuperado de https://scielo.conicyt.cl/ scielo.php? script $=$ sci_arttext $\&$ pid $=$ S 0 718-65682015000 $300008 \& \operatorname{lng}=\mathrm{es} \& \mathrm{nrm}=\mathrm{iso} \& \mathrm{tlng}=\mathrm{es}$

Linares, M. D. (2010). Prácticas comerciales fronterizas de las mujeres paraguayas paseras en la ciudad de Posadas, Argentina. Contribuciones Científicas GAEA, 22, 327-338.

Mora, J. M. (2016). África Fronteras: muros frente amenazas. bie3: Boletín ieee, (1), 23-39.

Ojeda, N. (2008). Reflexiones acerca de las familias transfronterizas y las familias transnacionales entre México y Estados Unidos. Frontera Norte, 21 (42), 7-30.

Popescu, G. (2011). Bordering and OrderingtheTwenty-first Century: Understanding Borders, Plymouth,Rowman and LittlefieldPublishers Inc.

Rodríguez, M. (2007). Las rutas del tráfico ilegal de migrantes hacia Estados Unidos de América. Labor de la marina ecuatoriana. Análisis de la normativa aplicable en los países de tránsito (México y Guatemala). FORO Revista de derecho 7, 225-50.

Vargas, A. (2013). Reinterpretando la diplomacia de los Pueblos. Trabajos y Ensayos. Revista del Master y Doctorado en Estudios Internacionales, 17 